\title{
Pregnancy in women with hypertrophic cardiomyopathy
}

\author{
P. G. Pieper • F. Walker
}

Published online: 5 December 2012

(C) Springer Media / Bohn Stafleu van Loghum 2012

\begin{abstract}
Hypertrophic cardiomyopathy (HCM) is increasingly being diagnosed in pregnant women. Women with HCM generally tolerate pregnancy well. The risk is however higher in women who are symptomatic before pregnancy or in those with severe left ventricular outflow tract obstruction. The incidence of arrhythmias does not appear to be increased during pregnancy and maternal mortality is low. Prior to conception, women with HCM should have a risk assessment as well as genetic counselling. During pregnancy beta-blockers should be continued and the judicious use of diuretics may be required to treat symptoms of dyspnoea. A vaginal delivery with regional anaesthesia is usually appropriate. Women should be managed by a specialist multidisciplinary team.
\end{abstract}

Keywords Pregnancy $\cdot$ Heart disease $\cdot$ Hypertrophic cardiomyopathy

\section{Introduction}

Hypertrophic cardiomyopathy (HCM) has a prevalence of approximately $0.2 \%$, which means there are an estimated 33,000 patients with HCM in the Netherlands. HCM is increasingly diagnosed in women of childbearing age due to more widespread use of echocardiography and familial

P. G. Pieper $(\bowtie)$

Department of Cardiology, University Medical Centre Groningen,

University of Groningen,

PO Box 30.001, 9700 RB, Groningen, the Netherlands

e-mail: p.g.pieper@umcg.nl

\section{F. Walker}

The Grown Up Congenital Heart Disease Unit,

The Heart Hospital, University College London

Hospitals (UCLH) NHS Trust,

London, UK screening programs. The majority of young women with heart disease, including HCM, wish to consider pregnancy and therefore obstetric admission is a common cause for hospitalisation in this patient population $[1,2]$. However, to date pregnancy outcome data for these patients are scarce.

HCM has recently been defined by the European Society of Cardiology (ESC) as increased ventricular wall thickness or mass in the absence of loading conditions (hypertension, valve disease) sufficient to cause the observed abnormality [3]. Metabolic diseases such as Anderson-Fabry disease and glycogen storage disease, as well as cardiac amyloid, are therefore now classified as HCM. However, most pregnant women with HCM have mutations of sarcomere protein encoding genes that are responsible for the disease, implying an autosomal dominant inheritance [4]. Around $70 \%$ of HCM patients have at least 1 affected family member, but sporadic mutations are also known to occur. Myocardial disarray and fibrosis are the histological hallmarks of the disease. Both the extent and localisation of the hypertrophy are variable with asymmetric septal hypertrophy being the most common phenotype. Left ventricular outflow tract (LVOT) obstruction is present in approximately $70 \%$. The diagnosis is primarily made by echocardiography (Fig. 1), although cardiac magnetic resonance imaging has an additional role, particularly if the hypertrophy is isolated to the LV apex, and it can also diagnose the presence and severity of myocardial fibrosis using gadolinium injection.

The phenotypic expression of the disease is very heterogeneous, both in terms of the age when it becomes manifest and its severity. The clinical course can range from a lifelong asymptomatic status to one where individuals are highly symptomatic and functionally limited with heart failure and malignant arrhythmias. Though many female mutation carriers do not have manifest disease at fertile age, early presentation with clinical HCM also occurs $[5,6]$.

Diastolic left ventricular dysfunction is invariably present and some patients also develop systolic dysfunction later in 
Fig. 1 Hypertrophic cardiomyopathy is primarily diagnosed by echocardiography. Apical 4-chamber view, systolic frame, showing hypertrophied, echogenic interventricular septum (S), systolic anterior motion of the anterior mitral valve leaflet (arrow), dilated left atrium (LA), left ventricle (LV)

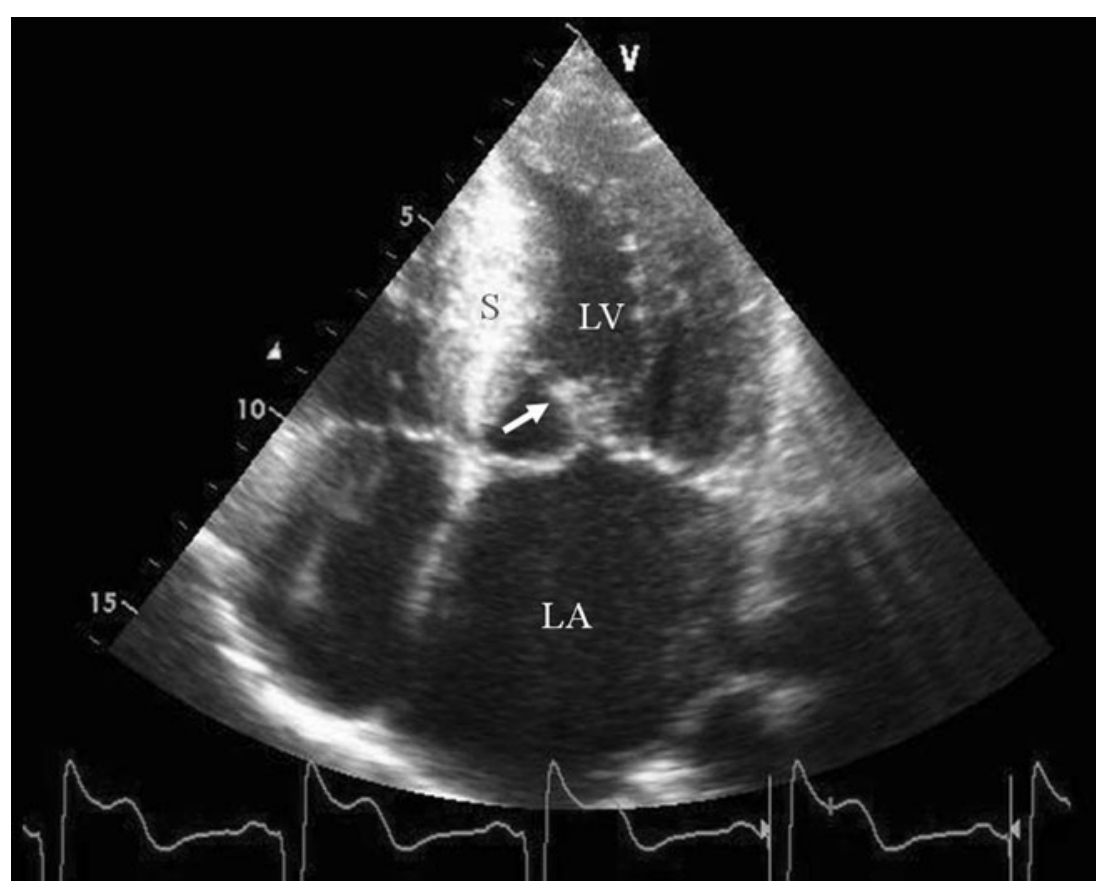

the disease course. The LVOT obstruction is often associated with mitral regurgitation, mostly due to systolic anterior motion (SAM) of the mitral valve, although intrinsic abnormalities of the mitral valve apparatus also contribute. Dyspnoea and chest pain are the most frequent symptoms and relate to the pathophysiological impact of the diastolic dysfunction, LVOT obstruction, mitral regurgitation, and myocardial ischaemia. Atrial fibrillation is the most common arrhythmia and it is poorly tolerated with an associated high risk of thromboembolism. There is an increased risk of sudden death, particularly in those with a family history of sudden death, symptoms of syncope, ventricular tachycardia, blunted blood pressure response on exercise and severe hypertrophy. In these patients, an implantable cardioverter defibrillator (ICD) may reduce the risk of sudden death [7-9].

Pharmacological therapy is indicated in symptomatic patients. Beta-blockers and verapamil are commonly used and disopyramide may be of help in those patients with severe LVOT obstruction. Diuretics may be needed to treat symptoms of dyspnoea but doses should be kept to a minimum. For rhythm control amiodarone is often needed and in patients troubled by atrial fibrillation, oral anticoagulation with warfarin is warranted. When symptoms persist despite medical therapy other treatment measures such as surgical myectomy or alcohol septal ablation can be considered.

\section{Pathophysiology related to pregnancy}

During pregnancy, plasma volume and cardiac output increase. The increase in cardiac output in the first and second trimesters is achieved by a larger stroke volume, while later in pregnancy there is an increase in heart rate. The additional volume load of pregnancy causes enlargement of the ventricular cavity, which theoretically might reduce the LVOT obstruction; however, the increased cardiac output tends to counteract this effect and the LVOT gradient will increase with advancing gestation. The same volume loading increases distension of the left atrium and thereby risk of atrial fibrillation. In the context of diastolic disease, the volume changes and increased heart rate are not well tolerated, aggravating symptoms of dyspnoea and lowering the threshold for developing left heart failure.

At the time of delivery, cardiac output increases further secondary to auto-transfusion of blood from the contracting uterus and increased catecholamine levels. There is also an increase in heart rate secondary to blood loss, pain and stress, while the expulsive efforts during delivery tend to diminish venous return. All of these physiological changes lead to an increase in LVOT gradient and shorten the diastolic filling period, therefore increasing the risk of pulmonary oedema.

\section{Risk assessment}

Only a limited number of pregnancies in women with HCM have been described in the literature [10]. The data that are available demonstrate that pregnancy is generally well tolerated [11, 12]; however, a subset of patients do experience significant complications [10].

To identify patients at increased risk a pre-conceptual review is recommended $[9,11,13]$. The ESC guidelines on the management of cardiovascular disease during pregnancy 
advise that a risk assessment is performed using the modified World Health Organization (WHO) classification. HCM is considered a WHO class II or III risk lesion, implying there is a moderate risk of morbidity in most women and potentially a significant risk for some [11]. In saying that, pregnancy is contraindicated in a minority of women, for example if there is significant impairment of systolic function $(\mathrm{LVEF}<40 \%$ ) or if there is severe symptomatic LVOT obstruction. In the latter group pregnancy may be possible after relief of obstruction by surgery or alcohol septal ablation.

To aid the pregnancy risk assessment, all women should be assessed clinically and undergo echocardiography and exercise tolerance testing $[9,11]$. Salient features to note on echo are measures of diastolic function, systolic function, localisation and severity of hypertrophy, outflow tract gradients at rest and with provocation, cavity dimensions and gradients, SAM and degree of mitral regurgitation and left atrial dimensions. On exercise the blood pressure response and maximum workload are important.

Previous studies in populations of women with both acquired and congenital heart disease have identified several predictors for cardiac complications during pregnancy. Amongst these are New York Heart Association class III or IV, left ventricular outflow tract obstruction and pre-pregnancy arrhythmias [2, 14]. However, these studies contained only a small number of women with HCM. Therefore, diseasespecific series also prove helpful when assessing risk. Autore et al. described pregnancy outcome in 49 women with HCM. Prior to pregnancy, 12 women were symptomatic and in 5 of these there was worsening of their clinical status ( $42 \%$ ) (either shortness of breath with minimal effort or pulmonary congestion). Of the 28 previously asymptomatic women only 1 developed symptoms $(4 \%)(p<0.01)[15]$.

Thaman et al. [16] reported on 271 pregnancies in 127 women. They also found a relationship between symptoms (mainly dyspnoea) before and during pregnancy, whereby of the $28.3 \%$ reporting cardiac symptoms during pregnancy, $90 \%$ had been symptomatic prior to pregnancy.

In the study by Avila et al. a relationship between symptoms before pregnancy and pregnancy outcome was not found. This study does, however, report a high percentage of heart failure $(30.3 \%)$, particularly during the third trimester, although details of LV function and LVOT obstruction severity are not given [17].

Two other studies describe lower rates of heart failure $[15,16]$ and in one report heart failure only occurred in the context of depressed left ventricular function [18].

In the study by Autore, clinical deterioration occurred more often in women with LVOT obstruction (peak gradient $>30 \mathrm{mmHg}$ ) than in women without obstruction ( $25 \%$ versus $11 \%)$; however, the difference was not statistically significant [15]. In Avila's study there was a small but significant increase in LVOT gradient in pregnancy compared with pre-pregnancy $(p<0.05)$, but those with outflow obstruction did not have more complications [17]. One retrospective study also found that LVOT obstruction was not a predictor of complications [16].

Maternal mortality is low in the few prospective studies that are available $[14,15,17]$. Combining these series there were a total of 71 women and 2 died during pregnancy; however, both were considered at very high risk and had been counselled against pregnancy.

Pregnancy does not appear to aggravate arrhythmias; all patients in the studies by Avila and Thaman who experienced syncope, palpitations or documented arrhythmia had experienced similar symptoms or events before pregnancy. Therefore, arrhythmias pre-pregnancy are probably predictive of arrhythmias during pregnancy, which is consistent with the literature concerning patients with other heart diseases $[2,14,16,17]$. Of note, in Avila's series, the occurrence of arrhythmias was similar in a pregnant and a nonpregnant group of women with HCM.

In 9 pregnancies in 8 women with HCM and an ICD in situ, there were 2 episodes of paroxysmal arrhythmia with one supraventricular tachycardia and one non-sustained ventricular tachycardia. These tachycardias were detected by the ICD but no therapy was delivered. One other patient had 2 episodes of sustained ventricular tachycardia which were terminated by anti-tachycardia pacing [18].

Thromboembolic complications have also been described $[17,18]$.

In conclusion, the current data on pregnancy outcome in women with HCM are scarce. Available data suggest that the
Table 1 Pre-pregnancy risk assessment in women with hypertrophic cardiomyopathy

\begin{tabular}{lll}
\hline & & Reference \\
\hline Risk assessment & & \\
Modified WHO classification & II or III & {$[11]$} \\
Predictors of increased risk & Symptoms pre-pregnancy & {$[2,14-16]$} \\
& LVOT obstruction (probably) & {$[2,14,15]$} \\
& Arrhythmias pre-pregnancy predict & {$[2,14,16,17]$} \\
& recurrence of arrhythmias & \\
& Impaired left ventricular function may & {$[18]$} \\
& be associated with heart failure & \\
\hline
\end{tabular}


risk of pregnancy is very much dependent on pre-pregnancy clinical status. The risk would appear to be higher in symptomatic patients, those with a history of arrhythmias, significant LVOT obstruction, or impairment of left ventricular systolic function. Severe complications are, however, rare. When discussing maternal risk with the future mother, the risk of disease recurrence in the offspring should be included and genetic counselling should be offered [12].

Risk assessment is summarised in Table 1.

\section{Management during pregnancy and delivery}

As per the ESC guidelines for the management of cardiovascular diseases during pregnancy [11], women with HCM who are in WHO class II should be reviewed by a cardiologist with clinical examination and echo assessment each trimester. For women in WHO class III more frequent follow-up, monthly or bimonthly, is warranted. These women should be managed in a specialist centre by a multidisciplinary team [11]. Follow-up during pregnancy should focus on the development of symptoms especially dyspnoea, arrhythmias, aggravation of outflow obstruction, and diastolic and systolic ventricular function.

Women taking beta-blockers prior to pregnancy should continue them during pregnancy and any woman who becomes symptomatic during pregnancy should start a beta-blocker and be prescribed diuretics if needed $[9,11]$. The ESC guidelines also recommend that a beta-blocker should be considered if there is more than mild LVOT obstruction or a septal thickness of $>15 \mathrm{~mm}$, in order to reduce the risk of pulmonary congestion [11].

Beta-blockers are also helpful for controlling atrial fibrillation and reducing the risk of ventricular arrhythmias. Metoprolol is the preferred beta-blocker since there is ample experience of its use in pregnancy; however, bisoprolol may also be used. Following birth, the infant should be monitored for bradycardia and hypoglycaemia.

One study has shown foetal growth retardation with maternal beta-blocker use; in this study, however, atenolol was used at supra-normal doses, therefore with standard dosing the risk of foetal growth retardation is probably low. However, atenolol is best avoided. Verapamil can be used during pregnancy, but caution is necessary due to the risk of foetal atrioventricular block. Amiodarone should not be used unless absolutely necessary, as it can induce foetal thyroid disorders. Sotalol can be used during pregnancy. Ultimately, any medication which is harmful to the foetus should not be withheld in case of maternal emergency, as maternal health is the priority. Atrial fibrillation is poorly tolerated and requires prompt cardioversion to restore sinus rhythm, either medically or electrically [11]. Electrical cardioversion is considered to be safe during pregnancy, but continuous foetal heart rate monitoring and the possibility for emergency caesarean section should be provided when the foetus is at a viable age [19].

By no later than the end of the second trimester a delivery plan should be in place, which should be the result of a joint discussion of the multidisciplinary team $[10,20]$. The minimal team requirements are an obstetrician, cardiologist and anaesthetist.

Vaginal delivery is usually preferred and caesarean delivery is reserved for obstetric indications [11]. Epidural anaesthesia is not routinely necessary in low-risk, asymptomatic HCM patients. Epidural and spinal anaesthesia must be administered cautiously in women with severe LVOT obstruction due to the consequent vasodilatation and hypotension and single-shot spinal anaesthesia should be avoided. However, when an epidural or spinal anaesthetic is administered judiciously, the benefits of pain reduction and reduced sympathetic stimulation may outweigh the disadvantages of these regional techniques [10, 11, 21]. Heart rhythm and blood pressure should be monitored but a pulmonary artery Swan-Ganz catheter is best avoided as it increases the risk of thromboembolism and arrhythmias, with little proven benefit $[11,21]$. Non-invasive monitoring of cardiac filling and LVOT obstruction can be effectively accomplished by transthoracic echocardiography when required [22]. Oxytocin should be given only as a slow

Table 2 Recommendations for the management of women with hypertrophic cardiomyopathy before and during pregnancy

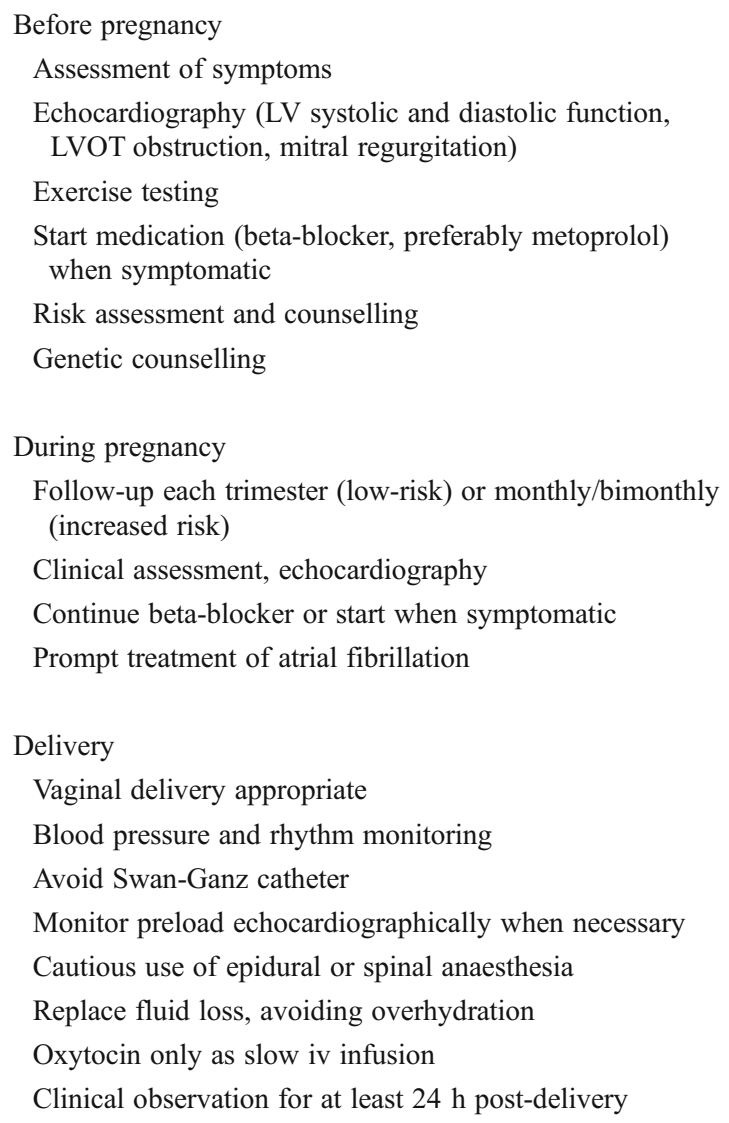


intravenous infusion. The dose should be kept to a minimum (i.e., 5 units) to avoid the side effects of hypotension, tachycardia and arrhythmia. Blood loss should be replaced to avoid a decrease in preload although fluid overload must also be avoided. The fluid shifts associated with delivery and immediately afterwards increase the risk of pulmonary oedema, therefore close observation and monitoring should be continued for at least $24 \mathrm{~h}$. The management of pregnant women with HCM is summarised in Table 2.

\section{Conclusions}

HCM is usually well tolerated in pregnancy but those with prior symptoms or arrhythmias tend to have a worsening of symptoms during pregnancy. The majority of these patients, however, can be managed medically with good effect. A small subset of women with severe LVOT obstruction are at increased risk but with expert management by a specialist multidisciplinary team, a good outcome can be expected for the most.

\section{References}

1. Verheugt CL, Uiterwaal CSPM, van der Velde ET, et al. The emerging burden of hospital admissions of adults with congenital heart disease. Heart. 2010;96:872-8.

2. Drenthen W, Boersma E, Balci A, et al. On behalf of the ZAHARA investigators. Predictors of pregnancy complications in women with congenital heart disease. Eur Heart J. 2010;31:2124-32.

3. Elliott P, Andersson B, Arbustini E, et al. Classification of the cardiomyopathies: a position statement from the European Society of Cardiology working group on myocardial and pericardial diseases. Eur Heart J. 2008;29:270-6.

4. Christiaans I, Nannenberg EA, Dooijes D, et al. Founder mutations in hypertrophic cardiomyopathy in the Netherlands. Neth Heart J. 2010;18:248-54.

5. Christiaans I, Birnie E, Bonsel GJ, et al. Manifest disease, risk factors for sudden cardiac death, and cardiac events in a large nationwide cohort of predictively tested hypertrophic cardiomyopathy mutation carriers: determining the best cardiological screening strategy. Eur Heart J. 2011;32:1161-70.
6. Van den Wijngaard A, Volders P, van Tintelen JP. Recurrent and founder mutations in the Netherlands: cardiac Troponin I (TNNI3) gene mutations as a cause of severe forms of hypertrophic and restrictive cardiomyopathy. Neth Heart J. 2011;19:344-51.

7. Ommen SR. Hypertrophic cardiomyopathy. Curr Probl Cardiol. 2011;36:409-53.

8. Ho CY. Hypertrophic cardiomyopathy in 2012. Circ. 2012;125:14328.

9. Gersh BJ, Maron BJ, Bonow RO, et al. 2011 ACCF/AHA guidelines for the diagnosis and treatment of hypertrophic cardiomyopathy. J Thorac Cardiovasc Surg. 2011;142:e153-203.

10. Krul SPJ, Smagt JJ, van den Berg MP, et al. Systematic review of pregnancy in women with inherited crdiomyopathies. Eur J Heart Fail. 2011;13:584-94.

11. Regitz-Zagrosek V, Blomstrom Lundqvist C, Borghi C, et al. ESC guidelines on the management of cardiovascular diseases during pregnancy of the European Society of Cardiology. Eur Heart J. 2011;32:3147-97.

12. Van Langen IM, Arens Y, Baars H, et al. ICIN working group on Hereditary Heart Diseases. Genetic diagnostics and genetic counselling in Hypertrophic Cardiomyopathy (HCM). Neth Heart J. 2010;18:144 59.

13. Pieper PG. Pre-pregnancy risk assessment and counseling of the cardiac patient. Neth Heart J. 2011;19:477-81.

14. Siu SC, Sermer M, Colman JM, et al. Prospective multicenter study of pregnancy outcomes in women with heart disease. Circulation. 2001;104(5):515-21.

15. Autore C, Conte MR, Piccinno M, et al. Risk associated with pregnancy in hypertrophic cardiomyopathy. J Am Coll Cardiol. 2002;40:1864-9.

16. Thaman R, Varnava A, Hamid MS, et al. Pregnancy related complications in women with hyprtrophic cardiomyopathy. Heart. 2003;89:752-6.

17. Avila WS, Amaral FMC, Ramires JAF, et al. Influence of pregnancy on clinical course and fetal outcome of women wit hypertrophic cardiomyopathy. Arq Bras Cardiol. 2007;88:423-8.

18. Schuler PK, Herrey A, Wade A, et al. Pregnancy outcome and management of women with an implantable cardioverter defibrillator: a single centre experience. Europace 2012; doi:10.1093/ europace/eus 172 .

19. Tromp CHN, Nanne ACM, Pernet PJM, et al. Electrical cardioversion during pregnancy: safe or not? Neth Heart J. 2011;19:134-6.

20. Pieper PG. The pregnant woman with heart disease: management of pregnancy and delivery. Neth Heart J. 2012;20:33-7.

21. Walker D, Kaur N, Bell R, et al. Hypertrophic obstructive cardiomyopathy and pregnancy: University College London Hospital experience. Minerva Anestesiol. 2007;73:485-586.

22. Ferguson EA, Paech MJ, Veltman MG. Hypertrophic cardiomyopathy and caesarean section: intraoperative use of transthoracic echocardiography. Int J Obst Anesth. 2006;15:311-6. 